\title{
De l'infra-conceptuel à des données à horizon de pertinence focalisé
}

Sylvie Leleu-Merviel

\section{(2) OpenEdition}

1 Journals

Édition électronique

URL : http://journals.openedition.org/questionsdecommunication/420

DOI : 10.4000/questionsdecommunication.420

ISSN : 2259-8901

Éditeur

Presses universitaires de Lorraine

Édition imprimée

Date de publication : 1 décembre 2010

Pagination : 171-184

ISBN : 978-2-8143-0056-9

ISSN : 1633-5961

\section{Référence électronique}

Sylvie Leleu-Merviel, « De l'infra-conceptuel à des données à horizon de pertinence focalisé », Questions de communication [En ligne], 18|2010, mis en ligne le 01 décembre 2012, consulté le 19 avril 2019. URL : http://journals.openedition.org/questionsdecommunication/420 ; DOI : 10.4000/ questionsdecommunication.420 


\section{$>$ ÉCHANGES}

SYLVIE LELEU-MERVIEL

Laboratoire des sciences de la communication

Université de Valenciennes et du Hainaut-Cambrésis

sylvie.merviel@univ-valenciennes.fr

\section{DE L'INFRA-CONCEPTUEL À DES DONNÉES À HORIZON DE PERTINENCE FOCALISÉ}

Résumé. - Les propositions que Patrick Charaudeau avance dans « Pour une interdisciplinarité «focalisée » dans les sciences humaines et sociales » sont mises en dialogue avec les travaux de Mioara Mugur-Schächter qui explicitent et organisent la genèse et la structure du contenu de l'entier volume du conceptualisé, en particulier de la connaissance scientifique. Partant de l'infra-conceptuel, qui se présente comme un magma de perçus indistincts, les processus d'élaboration de représentations transforment les perçus en entités signifiantes. Des procédures agréées de légitimation permettant une normalisation du sens conduisent à des significations, c'est-à-dire du sens négocié et partageable, détaché de son contexte, communicable, enseignable, apte à circuler, dès lors institué en objet social. En montrant comment s'entrelacent des concepts, des opérations, des données factuelles, une sémantique symbolique et une syntaxe qui assurent la communicabilité, le « canon descriptionnel » instauré met en relief les diverses composantes d'un « horizon de pertinence » que se doivent de partager des disciplines cherchant à se construire des espaces interdisciplinaires « focalisés ».

Mots clés. - Horizon de pertinence, représentations, interdisciplinarité, communication, données, faire-sens, signifiance, interprétation. 
Texercice de commentaire est difficile. En effet, comment discuter de façon pertinente un texte dont, en toute rigueur, chacune des parties nécessiterait analyse fine? Et qu'il est présomptueux de maintenir sa détermination à s'y risquer lorsque le texte en question commence par ces mots : « Comment prétendre traiter une question aussi vaste et complexe que l'interdisciplinarité quand, d'un côté, Claude Lévi-Strauss [...] invitait, au nom de la méthode du bricolage, à établir des connexions entre l'anthropologie, la linguistique, la littérature, l'art, la psychologie, le droit, la religion, etc., de l'autre, Edgar Morin incite, au-delà même de la transdisciplinarité, à « écologiser les disciplines » en tenant compte de «tout ce qui est contextuel y compris des conditions culturelles et sociales » et en adoptant parfois un point de vue 《 métadisciplinaire » » (Charaudeau, 2010 : 196). Décidément, la tâche est ardue, et ni le sujet traité ni les références écrasantes ne contribuent à la rendre plus légère. Impossible dès lors d'éviter l'écueil de l'anecdotique (extraction d'une partie très réduite du matériau fourni et discussion de cette seule partie, peu ou prou décontextualisée). Le présent travail n'y dérogera pas.

\section{Communication?}

Et puisque voilà d'emblée cette proposition condamnée à l'anecdotique, jouons ce travers jusqu'au bout de ses possibilités. Voici une petite saynète proposée comme support à la réflexion. Soit une personne A, dotée de tous les attributs nécessaires à ce qu'elle puisse légitimement être qualifiée de " parfaite » (attributs qu'on se gardera bien de détailler ici). A pénètre pour la première fois dans le bureau d'une personne B, qu'elle ne connaît pas, mieux que pas mal elle aussi. Aucune parole, aucun geste ne s'échangent.Y a-t-il communication? À n'en pas douter, instantanée, simultanée et parfaitement symétrique. II y a donc eu communication sans « acte de communication », puisque Patrick Charaudeau (ibid. : $216-217$ ) nous dit :

« On soutiendra l'hypothèse que tout acte de communication - qu'il s'agisse de communication interpersonnelle privée ou collective publique - se réalise toujours entre un sujet locuteur', et un sujet destinataire-récepteur-interprétant (qui peut devenir à son tour sujet locuteur, de façon symétrique), entre lesquels transite un certain acte de langage porteur de sens ayant une certaine forme ${ }^{2} \gg$.

Bien étrange concept à saisir que cette communication qui s'impose en totale déconnexion avec les faits ou actions permettant de la qualifier comme telle - au sens prescrit par Patrick Charaudeau tout au moins. Poursuivons notre histoire. A se déshabille. N'ayez crainte, rien d'érotique à cela, ce n'est pas une séquence torride qui débute, mais un examen médical, car B est médecin. À l'aide de diverses

\footnotetext{
«Locuteur » étant entendu au sens générique de tout sujet produisant un acte de langage oral ou écrit.

${ }^{2}$ Oublions ici le dédoublement des sujets du langage en identité sociale et discursive.
} 
méthodes (écoute à l'aide d'un stéthoscope, palpations, etc.), il va prélever sur ce corps des indices précis lui permettant d'élaborer un diagnostic. B aura presque totalement oublié la « perfection » première de ce corps, pour se concentrer sur le fait que son apparence dissimule une autre qualification : il est « malade ». La personne A a-t-elle communiqué - au sens prescrit précédemment? Pas le moins du monde. En revanche, elle a servi de support à une recherche d'information exploratoire qui a permis l'émergence d'une signifiance nouvelle : un diagnostic, pour l'instant encore hypothétique. L'affaire ne va pas bien du tout pour A : c'est grave! Voici son corps nu soumis à un examen par scanner, technique d'imagerie médicale qui, rappelons-le,

« consiste à mesurer l'absorption des rayons $X$ par les tissus puis, par traitement informatique, à numériser et enfin reconstruire des images 2D ou 3D des structures anatomiques. Pour acquérir les données, on emploie la technique d'analyse tomographique ou « par coupes », en soumettant le patient au balayage d'un faisceau de rayons $X »^{3}$.

B scrute l'image formée par la recomposition de ces différentes coupes, qui lui permet de conforter son diagnostic. La personne A, bombardée qu'elle est de rayons $X$, ne communique toujours pas davantage, la pauvre. $B$ a complété par cette nouvelle image de A l'information disponible sur la maladie présumée. À ce stade, $B$ a souvent totalement occulté $A$ au profit de sa maladie, dont il se construit progressivement une représentation de plus en plus fine. II se trouve que la personne A est ce qu'on appelle un « cas clinique » inédit. Elle est rentrée chez elle, mais elle ne sait pas que ses résultats de scanner ont été transmis à toute une communauté qui en débat, conformément au principe de l'« étude de cas ». Revenons à Patrick Charaudeau (ibid. : 217) qui explique :

\begin{abstract}
«Lorsqu'il s'agit d'une communication collective qui se déploie dans un espace public à l'aide de divers supports de transmission, on dira que la communication se réalise entre une instance de production du langage ${ }^{4}$, et une instance de réception-interprétation (qui peut devenir à son tour instance de production mais de façon asymétrique), entre lesquels transite un certain produit porteur de sens ayant diverses formes selon le système de signes (verbal, iconique, gestuel) qui le configure. Ces instances sont constituées par des acteurs qui agissent, qui pensent, qui parlent et qui se trouvent les uns vis-à-vis des autres dans diverses relations selon les statuts et les rôles qu'ils ont à tenir. Certains de ces acteurs tiennent des rôles de producteur de discours (par exemple, pour les médias : direction de l'organe d'information, rédacteurs en chef, journalistes, etc.), d'autres tiennent des rôles de récepteur (par exemple, lecteurs, auditeurs, téléspectateurs), les uns et les autres étant tenus par certaines contraintes tant du côté de la production que du côté de la réception. Du même coup, ces acteurs sont amenés à se représenter la place qu'ils occupent et qu'est censé occuper l'autre, la finalité de l'échange dans laquelle ils sont impliqués et les conditions qui président à cet échange ».
\end{abstract}

Dans notre cas, peut-on circonscrire précisément une instance de production conforme aux termes de cette définition? Est-ce B qui a fait circuler le cas à la communauté? Ou est-ce la machine, qui a fourni l'image? Ou encore le corps

\footnotetext{
3 Wikipédia, rubrique «Tomodensitométrie ».

${ }^{4}$ «Langage » est ici entendu dans un sens large comme tout système de signes permettant de communiquer.
} 
de A, dont c'est une représentation? Et tous les spécialistes qui débattent autour du cas ne sont-ils pas à la fois producteurs et récepteurs, dans un paradigme de construction collaborative, réciproque, simultanée et symétrique, entre pairs de statut égal? Cette historiette n'a pas pour but - bien entendu - de disqualifier le travail de Patrick Charaudeau, mais de montrer qu'il possède son horizon de pertinence qu'il convient de ne pas oublier, son « lieu géométrique » qu'il circonscrit lui-même : pour faire simple et donc forcément réducteur, celui d'une communication mesurée à l'aune des actes de langage et recourant à l'analyse de discours comme point de vue prioritaire - point de vue d'un sémiologue, analyste du discours ayant traversé les sciences du langage de l'époque structuraliste à nos jours affirme-t-il dès l'introduction de son texte. Elle montre aussi que le regard propre aux sciences de l'information et de la communication peut être différent, élargi notamment par le biais de ce qui constitue son caractère spécifique: la prise en considération simultanée - et presque indistincte, pourrait-on dire parfois - de l'information et de la communication, en intrication étroite et sans tentative (ni même tentation) de décorrélation préliminaire et/ ou arbitraire. Dès lors, est invalidée l'hypothèse posant un modèle d'analyse de la communication à partir de trois lieux de pertinence séparés : le lieu des conditions de la production, le lieu des conditions de la réception, et le lieu de réalisation de l'acte de communication comme produit fini.

\section{Horizon de pertinence?}

L'« horizon de pertinence » scientifique - plutôt que « principe ou lieu de pertinence » - est apparu « spontanément» au paragraphe précédent. II se présente comme un savant mixage de l'« horizon d'attente » de Hans R. Jauss (1978) et de la théorie de la pertinence de Deirdre Wilson et Dan Sperber (1989). II assemble les divers items énumérés par Patrick Charaudeau : cadre conceptuel de référence, théories, catégories, modes de raisonnement, procédures d'analyse. II n'est pas inutile d'insister sur le fait que la détermination de l'horizon de pertinence est toujours indispensable. On pourrait croire qu'elle ne s'impose que pour les disciplines récentes, trop jeunes pour que leurs assises scientifiques soient totalement assurées. On aurait tort. La suite de cet « échange » va beaucoup recourir aux travaux de la physicienne Mioara Mugur-Schächter, qui a fondé et dirigé le laboratoire de mécanique quantique et structures de l'information à l'université de Reims Champagne-Ardenne. Dans son ouvrage L'infra-mécanique quantique. Une révolution épistémologique révélée dans les descriptions de microétats (Mugur-Schächter, 2009 : 49-52), elle montre que les deux notions fondamentales et incontournables de la physique, celles que l'on pourrait croire à jamais impossibles à réfuter, l'espace et le temps, sont des construits relativisés dont l'horizon de pertinence est limité :

« Selon Kant, l'espace et le temps ne sont pas du réel physique, ni des "propriétés" du réel physique. Ce sont des réceptacles innés où se logent les perceptions et les connaissances 
De l'infra-conceptuel à des données à horizon de pertinence focalisé

humaines concernant le réel physique. L'épistémologie kantienne opère une transmutation des absolus physiques de l'espace et du temps newtoniens, en traits du psychisme humain [...]. Considérons d'abord les deux théories de la relativité d'Einstein. Commençons avec les vues concernant l'espace-temps. Selon l'entière physique classique l'espace et le temps sont des donnés absolus. Tandis que les deux théories einsteiniennes de la relativité - élaborées elles aussi en tant que disciplines macroscopiques - assignent une validité relativisée aux résultats des mesures de distances et de durées [...]. Quant aux représentations de la mécanique quantique, elles concernent foncièrement des entités microscopiques, étrangères à la physique de Newton et à la philosophie kantienne et néo-kantienne. Dans cette théorie les concepts d'espace et de temps sont atteints d'un degré notable de désagrégation et évanescence, à cause de la nature primordialement probabiliste des descriptions ».

Sous ces quelques mots d'apparence anodine se cache la révolution épistémologique annoncée par le titre de l'ouvrage - très mal acceptée, du reste, par la communauté des sciences physiques. Car, comme l'affirme Mioara Mugur-Schächter (ibid. : 48),

\begin{abstract}
« la pensée physique classique a totalement occulté la question épistémologique. Elle a laissé un trou épistémologique qui prolonge le vide philosophique concernant l'existence même des consciences individuelles avec leurs subjectivités et leurs formes a priori. Corrélativement, la physique classique pose que l'on découvre les lois "objectivement vraies" du réel physique; que, au fur et à mesure que la physique progresse, elle nous rapproche de manière asymptotique de la connaissance du réel physique tel qu'il est en lui-même vraiment, objectivement, en entendant par ces mots : indépendamment de toute perception ou action cognitive humaine. La physique classique ne se soucie pas de spécifier comment les physiciens - des hommes qui pour connaitre agissent nécessairement à travers leurs consciences individuelles liées à leurs corps - peuvent arriver à découvrir les lois objectives de la réalité physique telle qu'elle est indépendamment de toute conscience humaine, de toute perception ou action cognitive humaine ».
\end{abstract}

Cette citation confirme l'abolition pure et simple d'une « réalité physique » qui serait « vérité scientifique » connaissable en soi, concept sur lequel s'appuient pourtant avec autorité et certitude les sciences dites « dures ».

\title{
De ce qui « existe » et de la connaissance
}

Mioara Mugur-Schächter (ibid.: 46) concrétise cette idée de la manière suivante :

« II est clair qu'une fourmi perçoit le réel - quoi que cela peut vouloir dire - autrement qu'un homme ou qu'un aigle; que si je regarde la tête d'un chaton sous une grande loupe, elle m'apparait comme celle d'un tigre, cependant qu'au seul toucher, sa patte continue de me sembler minuscule; que - selon les modèles de la microphysique actuelle - pour un neutron qui serait doté d'une capacité de perception consciente de type humain, le corps humain dont il fait partie apparaitrait comme un nuage de petits maxima de densité de substance grave très éloignés les uns des autres; etc. Toutes ces relativités aux modes de percevoir crèvent l'entendement dès qu'on analyse la genèse des perceptions et l'on met en jeu les vues de la science actuelle. Le réel en soi ne peut être de toutes ces façons différentes à la fois. II ne peut que, soit être tout court, caché dans une stricte absence de toute apparence, donc de toute connaissabilité, soit paraître tel ou tel, à travers telle ou telle grille de perception ou de représentation et donc être connu à travers cette grille ». 
Il en va exactement de même de la personne A de notre petite histoire. Elle est «vue » successivement de quatre façons différentes : comme une forme humaine perçue naturellement via le regard d'un autre être humain, sans intermédiation appareillée - sauf si B porte des lunettes ou des lentilles, ce que l'histoire ne dit pas; comme un patient dont les symptômes sont expertisés via l'appareillage basique usuel - méthodologique et technique - de la médecine allopathique; comme une image tomodensitométrique définissant, grâce à un regard très spécifique généré par le biais d'une technologie sophistiquée, les contours d'une pathologie sévère; comme un cas clinique sujet à débats susceptibles d'enrichir la connaissance médicale spécialisée. Aucune de ces quatre vues n'est plus vraie que les autres; ou plus exactement, elles sont toutes aussi vraies, et simultanément vraies, mais répondent simplement à des regards différents. Perte définitive donc de la « vérité scientifique » en tant qu'absolu : le réel objectif tel qu'il est en luimême est transcrit également, aussi bien ou aussi mal, dans chacune de ces quatre vues, ainsi que dans une infinité d'autres. S'il peut paraître incongru dans un travail comme celui-ci, ce petit détour par la physique nous a au moins démontré que les choses ne sont pas plus « objectives 》 ou ancrées dans le réel en sciences dites exactes qu'ailleurs : les fondements épistémologiques de la production de connaissances, abondamment discutés en sciences humaines et sociales, posent question là aussi, et de la même manière, même si l'incontestable prédictibilité pratique acquise par les sciences dures renvoie souvent ces questions-là dans les enfouissements de l'inavoué. Ce résultat préserve d'absolutisations abusives. Pour reprendre l'un des exemples cité par Patrick Charaudeau (20 I 0), le concept de représentation n'est pas plus « exact» ni plus « vrai » en philosophie qu'il peut l'être en analyse du discours, en sociologie, en psychologie sociale ou en anthropologie, non plus qu'en mathématiques, en médecine, en informatique graphique, en sciences de l'information ou en esthétique. II est validé et ainsi rendu opératoire, dans chacun de ces cadres, par l'horizon de pertinence qui le circonscrit, et qui doit sans cesse être redéfini précisément, dès lors que l'on envisage une migration ou même le plus insignifiant glissement de sens le concernant. Autrement dit, ce n'est pas tant l'emprunt ou la migration de concept - qui est courant, comme on peut le constater - que la spécification des horizons de pertinence respectifs et la détermination de ce qui peut en être partageable qui compose le cœur d'une interdisciplinarité « focalisée ».

\section{Représentations partageables}

La notion d'horizon de pertinence est intimement tissée avec celle de représentation. Elle soutient la problématique liée à la manière dont se construisent et s'organisent les pensées, les connaissances et/ou les savoirs. Pour mieux la cerner, il convient de s'attarder sur la façon dont les connaissances sont élaborées et circulent, de la subjectivité stricte aux constructions intersubjectives. En effet, après l'abolition définitive du « réel en soi », la principale révolution 
opérée par Mioara Mugur-Schächter et qui est proposée ici à la réflexion, est de replacer la totalité des phénomènes connus et connaissables dans la relativité de leur perçu, c'est-à-dire dans le subjectif strict. Car tout système interprétant, au sens de Gregory Bateson (1972) - qu'il soit automate, animal ou humain -, évolue en interaction avec un environnement dont il perçoit certaines propriétés (« focalisées » et, de ce fait, pas toutes) à partir desquelles il fonde ses propres comportements et ses réactions en retour. L'appréhension du monde et l'aptitude à y survivre passent par l'expérience. Patrick Charaudeau (2010 : 209-210) ne dit pas autre chose, puisqu'il affirme qu'« on ne peut dissocier représentation et action, et donc activité cognitive, pratique sociale et conscience agissante des sujets. Ceux-ci sont dotés de la faculté de percevoir et d'évaluer les actions collectives, ce qui leur permet d'ajuster leurs conduites ». En outre, il rappelle que Gaston Bachelard oppose la « conceptualisation », activité rationnalisante qui produit la science, à la « rêverie », activité créatrice produisant une vision poétique du monde; mais il associe immédiatement ces deux activités en ce qu'elles sont à l'origine des principes organisateurs des conduites (Charaudeau, 2010 : 208). Selon Jean-Louis Le Moigne (1995: 71), « le sujet ne connaît pas de choses en soi - hypothèse ontologique - mais il connaît l'acte par lequel il perçoit l'interaction entre les choses ». Pour Edgar Morin (1994), notre seule réalité immédiate se réduit à notre représentation de la réalité au travers de l'expérience que nous en avons. Dans un premier stade de l'interaction, le perçu est globalisant, intuitif et dépourvu de toute caractérisation et/ou catégorisation. C'est ce que Mioara Mugur-Schächter dénomme l'infra-conceptuel, qui se présente comme un magma de perçus indistincts. La faculté de classement apparaît au deuxième degré de la représentation, après l'automatisme qui est le niveau 0 et la proto-représentation de niveau I, avec la capacité à catégoriser. Dans un précédent travail (Leleu-Merviel, 2003) dont une synthèse partielle est reprise ici, je proposais une hiérarchisation des capacités représentationnelles et montrait, à partir des travaux de Joëlle Proust (2003), que les animaux sont tout à fait capables de catégorisation. C'est par exemple ce que fait l'araignée qui sent vibrer sa toile. Les capteurs de vibration fournissent un stimulus sensoriel; celui-ci est analysé par un comparateur qui a mémorisé les « signatures » vibratoires des proies antérieures. L'araignée peut également garder en mémoire, en cas de prise multiple, les positions de plusieurs proies dans sa toile (ibid. : 29). Ce stade permet à l'araignée de diversifier les réponses en fonction de circonstances identifiées, mais par voie réflexe, sans possibilité de « décider » en autonomie d'une réponse parmi un échantillon de possibles. Par ailleurs, elle ne forme pas une représentation du monde sous forme d'objets indépendants : elle catégorise les perceptions de traits centrés sur sa propre réactivité, et qui occasionnent, par association, un certain type de réaction (selon qu'il s'agit de consommer ou de fuir). Une représentation «mentale » exige davantage. Ce n'est que lorsqu'apparaît la capacité de former des représentations « détachées 》 (leur contenu n'est pas centré sur la façon dont il va être utilisé) que le dispositif peut être qualifié de cognitif. La représentation détachée doit satisfaire deux principes: 
I. la condition d'évaluation : la représentation peut être vraie ou fausse, l'objet possède ou non telle propriété modale reconnue comme un invariant dans le monde;

2. le principe de généralité : l'entité doit pouvoir attribuer une propriété quelconque, parmi celles qui figurent dans son répertoire, à un objet quelconque.

La première propriété de la représentation détachée est la référence. Pour pouvoir former des représentations détachées, les canaux de traitement correspondants à diverses sollicitations sensibles doivent pouvoir opérer de manière spatialement cohérente, c'est-à-dire qu'ils doivent être interconnectés et calibrés pour s'accorder sur la région de l'espace concerné par un stimulus donné. Le sujet percevant - ou plutôt son cerveau - apparie alors plusieurs types de données sensorielles pour les exploiter ensemble, rapportant ainsi diverses propriétés indépendantes à un même objet. La référence est présente chez bon nombre d'animaux, notamment les mammifères, les serpents et les oiseaux. La seconde propriété de la représentation détachée est l'emploi de concepts. Leur usage suppose de catégoriser un objet ou une propriété, et d'en tirer les conséquences pour maintenant ou pour plus tard. Utiliser des concepts consiste à élaborer des propositions qui peuvent être vraies ou fausses - i.e. sémantiquement évaluables - et qui servent à produire des inférences. Les principales conditions de la représentation conceptuelle, c'est-à-dire l'abstraction, l'orientation et la représentation globale de l'espace, l'apprentissage par généralisation, la mobilisation dirigée vers un but, sont présentes chez un grand nombre d'animaux, en particulier les vertébrés. Cette étape correspond à l'apparition des premières formes de raisonnement pratique. La conceptualisation est la réponse apportée par l'évolution au problème de la surcharge. Un système perceptif reçoit un grand volume de données, dont une grande part est sans pertinence (bruit). Quant à la part utile (signifiante), elle ne peut l'être que si elle est correctement exploitée. Processus performant de compression, la conceptualisation convertit les données sensorielles en réduisant les entrées perceptives à des structures minimales cohérentes, permettant ainsi de schématiser. Le langage étend considérablement les capacités de la représentation conceptuelle en lui attribuant une dimension communicationnelle intersubjective (concepts partageables et dont la légitimité est évaluable ou réfutable par autrui). Le langage permet en outre d'élaborer des objets abstraits, ou des concepts d'ordre supérieur (comme le concept de concept). Grâce au développement de la mémoire, les données concernant un objet ou un événement peuvent être complétées ultérieurement, ou révisées. Cette indépendance entre temps et contenu du codage permet une appréciation flexible des conditions de l'action et favorise la planification. La question demeure ouverte, mais la plupart des spécialistes estiment que la capacité de prévoir l'avenir ou de planifier son action sans apprentissage préalable est inaccessible aux animaux non langagiers. Le langage est donc bien davantage que l'exploitation d'un code commun à l'émetteur et au récepteur: 
il suppose la compréhension de l'intention qui préside à l'envoi du message. Par ailleurs, la mise en concordance d'une sémantique symbolique et d'une syntaxe rend les propositions combinables, ce qui permet la production d'un ensemble ouvert (en principe infini) de messages nouveaux portant sur des états de choses indéterminés. Les systèmes de signaux animaliers peuvent avoir une syntaxe, comme le chant des oiseaux ou des baleines. Ils peuvent renvoyer sémantiquement à un fait extérieur ou à une propriété de l'émetteur, comme les cris d'alarme ou les cris territoriaux. Ils ne disposent jamais de toutes les propriétés articulées en cohérence. II n'y a pas de place pour l'invention de signaux radicalement nouveaux pour faire référence à des événements qui ne sont pas déjà au « répertoire » des individus de l'espèce considérée. Le tableau ci-dessous (lui aussi repris de Leleu-Merviel, 2003 :26), récapitule les cinq niveaux représentationnels et leurs caractéristiques principales - le lecteur est renvoyé au texte complet, ainsi qu'à l'ouvrage de Joëlle Proust (2003), pour une présentation plus détaillée.

Figure I. Tableau récapitulatif des cinq niveaux représentationnels

( $M$ : machine, $A$ : animal, $H$ : humain).

\begin{tabular}{|c|c|c|c|c|c|c|c|}
\hline & $\begin{array}{c}\text { Type de } \\
\text { représentation }\end{array}$ & $\begin{array}{l}\text { Type du } \\
\text { dispositif }\end{array}$ & Modalités et fonctions & $\begin{array}{l}\text { Niveau de } \\
\text { compétences }\end{array}$ & $M$ & A & $\mathrm{H}$ \\
\hline 0 & & Automatique & $\begin{array}{l}\text { Covariance avec une } \\
\text { variable extérieure }\end{array}$ & Réflexe & $*$ & * & * \\
\hline 1 & $\begin{array}{l}\text { Proto- } \\
\text { représentation }\end{array}$ & Asservi & $\begin{array}{c}\text { Covariance avec un écart } \\
\text { différentiel significatif d'une } \\
\text { variable extérieure }\end{array}$ & $\begin{array}{l}\text { Réaction } \\
\text { flexible }\end{array}$ & & * & * \\
\hline 2 & Catégorisation & $\begin{array}{c}\text { Adaptatif ou } \\
\text { personnalisable }\end{array}$ & $\begin{array}{l}\text { Classement des objets } \\
\text { et événements perçus et } \\
\text { mémorisation, capacité à } \\
\text { tirer parti de l'expérience } \\
\text { passée }\end{array}$ & $\begin{array}{l}\text { Réponses } \\
\text { diversifiées }\end{array}$ & * & * & * \\
\hline 3 & $\begin{array}{l}\text { Représentation } \\
\text { détachée }\end{array}$ & Cognitif & $\begin{array}{l}\text { Manipulation de concepts } \\
\text { détachés et inférences }\end{array}$ & $\begin{array}{c}\text { Raisonnement } \\
\text { pratique }\end{array}$ & & * & * \\
\hline 4 & $\begin{array}{l}\text { Communication } \\
\text { intersubjective }\end{array}$ & Langagier & $\begin{array}{c}\text { Concepts partageables } \\
\text { et dont la légitimité est } \\
\text { évaluable ou réfutable par } \\
\text { autrui, propositions aux } \\
\text { contenus combinables à } \\
\text { l'infini }\end{array}$ & Pensée & & & * \\
\hline
\end{tabular}

La faculté supérieure la plus spécifique à l'être humain réside donc sans conteste dans sa créativité, c'est-à-dire sa capacité à innover en faisant émerger des représentations novatrices et différenciées, totalement inédites. L'une de ses caractéristiques propres est aussi d'élaborer des représentations intersubjectives partageables, et donc communicables, légitimées et instituées en objets sociaux 
circulants. Mais la mise en circulation de représentations partagées implique des processus de légalisation des signifiés.

\section{De la légalisation des signifiés}

D'ailleurs, c'est un travail très important de la démarche scientifique, et qui distingue ses construits de ceux du langage naturel. En effet,

« il y a [...] une certaine finalité spontanée incorporée à tout langage naturel, un but incorporé de malléabilité des significations assurée par la possibilité de compositions de mots très diverses. Les langages courants ont besoin de certains flous, car au cours des processus de communication ceux-ci dotent l'expressivité d'élasticité, de richesse et de résilience. Mais pas n'importe quel flou [...]. La frontière d'un concept désigné par tel mot, bien qu'implicite, résiste au flou où la plonge un trompe-l'œil conceptuel. En outre, la recherche de la possibilité de moduler le sens d'un mot par le contexte s'affaiblit dans les langages spécialisés. Dans un langage formel réussi elle est même éliminée entièrement. Dans ce cas limite d'un langage spécialisé de logique formelle, le but est diamétralement opposé à celui de pouvoir moduler le sens d'un mot à l'aide de son contexte: on y cherche au contraire l'unicité stricte de la signification de chaque signe d'étiquetage, afin d'assurer aux trajets déductifs une rigueur parfaite, à l'abri de toute ambiguïté » (Mugur-Schächter, 2009 : 35-36).

L'élaboration d'un objet, qu'il soit concret ou abstrait, s'accomplit ainsi sur la base de propriétés qui émergent dans l'interaction et les perçus qui l'accompagnent, avec toute la relativité qui les caractérisent. En effet, « tout objet est foncièrement relatif aux grilles sensorielles et aux formes de conceptualisation introduites par la conscience où il s'est constitué. Notamment, il est relatif aux seuils de perception que comportent les grilles sensorielles mises en jeu. Si l'homme pouvait distinguer des dimensions spatiales de l'ordre de $10^{-10}$ d'un centimètre et des dimensions temporelles de l'ordre de $10^{-30}$ d'une seconde, les objets construits par les consciences humaines seraient tout à fait autres, ou au moins différents de ce qu'ils sont. Par exemple, une cuillère apparaitrait comme un nuage à bords mouvants. Et les objets formés par les appareils sensoriels d'autres espèces d'êtres, un oiseau, un insecte, sont en général différents des objets que forment les hommes » (ibid. : 37-38). Dans cette approche, le primat est rendu aux consciences qui construisent, plutôt qu'aux « faits objectifs 》. Les effets immédiatement perceptibles des interactions entre une conscience et son environnement s'élaborent comme des « apparences phénoménales 》. «Tels qu'ils émergent d'abord dans les consciences, ce sont encore des faits strictement subjectifs.Afin qu'ils deviennent communicables et donc susceptibles de consensus intersubjectifs et notamment scientifiques, les phénomènes doivent être soumis à certaines opérations de légalisation à la suite desquelles ils se trouvent objectivés. Selon Emmanuel Kant, l'objectivité scientifique consiste donc en consensus intersubjectifs concernant des apparences phénoménales qui au départ sont foncièrement subjectives, mais qui sont par la suite légalisées, notamment selon des méthodes scientifiques » (ibid. : 45). Cette approche n'est pas sans rappeler celle de François Rastier (2003) qui établit un renversement entre « sens » et 
De l'infra-conceptuel à des données à horizon de pertinence focalisé

« signification ». Le « sens » n'est pas de la « signification » altérée par le contexte individuel, situé et pragmatique. C'est l'inverse : la « signification » s'apparente à du « sens normalisé » (ibid. : 9), détaché de son contexte, généralisé, marqué ainsi par une certaine stabilité. Dès lors, elle devient transmissible, partageable, peut se communiquer et circuler, se construire donc en objet social. Les significations, en tant que résultat d'une normalisation, impliquent la présence de procédures publiques de légitimation, faisant l'objet d'un consensus social au moins partiel (c'est-à-dire agréées dans une communauté non réduite à un seul individu). D'un point de vue proprement linguistique, l'approche rejoint également la « théorie de la pertinence » de Dan Sperber et Deirdre Wilson, où les « représentations partagées », fondatrices du langage, reposent sur l'idée d'adhésion des membres d'un groupe à des valeurs communes qui feraient consensus pour qu'ils puissent communiquer.

\section{Les éléments de l'horizon de pertinence}

Sans du tout épuiser le sujet, une dernière question doit être abordée pour clore le tour d'horizon proposé : il s'agit des données factuelles et de leur lien avec les procédures qui s'y appuient dans l'élaboration des représentations. Toute discipline scientifique « s'affronte au problème du repérage des données pertinentes, de leur recueil, de leur classement selon certaines catégories, de leur analyse et de l'interprétation des résultats » écrit Patrick Charaudeau (2010: 204). Mais que sont exactement les « données »? Ayant aboli la notion de donnés absolues fournies par l'essence même du réel, donnés au sens « d'offerts à la saisie », « prêts à prendre », que deviennent donc les « données » dans cette approche? Et quand et comment sont-elles pertinentes? On commencera par accepter un postulat :

« Toute transposition d'un phénomène en termes communicables équivaut en essence à la construction d'une description : rien d'autre que des descriptions ne peut être connu d'une manière intersubjective non restreinte. Ni des entités factuelles extérieures à toute conscience, ni des phénomènes non décrits, ne peuvent être connus d'une manière intersubjective non restreinte. Ce qui n'est pas décrit peut être ressenti en un certain sens, mais ne peut pas être communiqué à toute distance et sans limitations, dans tous les détails et sans ambiguïtés (pas juste montré du doigt ou dessiné, dansé, crié ou gémi, etc.). Cette spécification est loin d'être triviale. Elle focalise l'attention sur l'importance primordiale du contenu et de l'agencement d'une description quelconque et notamment sur le degré et la qualité de la communicabilité que ce contenu et agencement assurent » (ibid. : 202).

Cette question mériterait à elle seule une longue et profonde analyse. II n'est pas certain que quelques sortes de phénomènes - les comportements humains par exemple - ne soient pas mieux communiqués dans une forme métaphorique telle une pièce de théâtre - que par le biais d'une description. Laissons agir le flou conceptuel que comporte le qualificatif de « restreint » pour désigner 
cette communicabilité là, et accordons-nous sur le fait d'accepter ce préliminaire comme un postulat.

« Les langages naturels avec leurs grammaires nous ont profondément conditionnés à supposer plus ou moins explicitement que ce qui, dans les descriptions, joue le rôle d'entité-objet-dedescription, préexiste aux descriptions en tant qu'objets tout court, qualifiés à l'avance par des propriétés qu'ils posséderaient à l'état déjà actuel et indépendamment de tout examen [...]. Quant aux opérations de qualification, selon la pensée classique telle qu'elle est reflétée par les grammaires et par la logique, tout le processus qui d'abord crée un qualificateur et ensuite crée les qualifications correspondantes est rétréci en un seul acte statique, presque passif, de simple détection sur une entité-objet préexistante, d'une propriété préexistant dans cette entité » (Mugur-Schächter, 2009 : 203-204).

Or, cette sorte d'évidence de ce que sont les propriétés et de la façon dont elles qualifient les phénomènes ou les événements est totalement réfutée par la méthode de conceptualisation relativisée (MCR). Elle « débute à des zéros locaux de conceptualisation, en y représentant la capture de fragments de substance physique purement factuelle, encore a-conceptuelle, qui par la suite sont traités comme une matière première pour des sémantisations progressives. Ces sémantisations constituent le cœur même de l'entière démarche » (ibid. 2006 : 25). La méthode MCR prône ainsi que dans tous les cas, l'observateurchercheur crée une entité-objet qui ne préexistait pas, souvent tout à fait indépendamment des opérations de qualification qui ne sont réalisées qu'ensuite. En outre, celles-ci sont doublement relatives : le mode de génération de l'entité-objet, en produisant le fragment de matière première support de la future connaissance, peut exclure certains examens ou en favoriser d'autres; les modes d'examen influencent également les qualifications produites. Les chaines d'opérations de conceptualisation commencent donc systématiquement par une extraction légalisée de fragments de pure factualité physique. Une biopsie pratiquée sur la personne $A$ de l'exemple initial illustre très concrètement ce type de « prélèvement » (mais n'en est qu'une instance parmi une infinité de possibles quant à cette seule personne A). Cependant, l'échantillon tissulaire ne dit rien en lui-même. II faut ensuite le qualifier à l'aide de qualificateurs construits explicitement pour assurer la communicabilité de leurs « résultats 》, donnant lieu à des consensus intersubjectifs répondant à des buts. Par exemple, le prélèvement sera soumis à tel type d'analyse pour détecter l'éventuelle présence de cellules cancéreuses. Mais d'autres « regards », appareillés différemment et donnant lieu à d'autres « mesures», pourront fournir d'autres qualifications : groupe sanguin, structure génomique, etc. Fort probablement, la biopsie ne produira pas la bonne entité-objet pour déterminer si la personne $\mathrm{A}$ a les yeux bleus. Cet exemple montre bien le « canon descriptionnel » à l'œuvre : saisie de fragments de substance a-conceptuelle, génération de l'entité-objet, choix explicite de propriétés ou aspects qualifiants répondant à des buts précis, méthodologie et appareillage - théoriques et/ou artefactuels - pour effectuer la qualification. Le « coup » tiré par ce canon descriptionnel produit les données. Ainsi apparaissent-elles désormais exactement contraires à du « naturel prêt à saisir », mais comme des construits façonnés par la méthode de construction, 
De l'infra-conceptuel à des données à horizon de pertinence focalisé

laquelle relève d'un choix cognitif - volontaire et totalement assumé dans la démarche scientifique, souvent réflexe dans la vie courante. C'est l'ensemble de ces éléments, maintenus ensemble en cohérence, qui constitue l'horizon de pertinence des représentations conceptuelles.

\section{Conclusion}

De ce qui précède, on déduit qu'il existe de nombreuses manières de ne pas se comprendre entre disciplines : extraire des fragments différents d'une même réalité sans s'apercevoir que ce ne sont pas les mêmes fragments; les examiner sur la base d'aspects qualifiants différents, en ignorant qu'ils sont différents; procéder à la qualification par des méthodologies ou via des outils qui n'ont strictement rien à voir les uns avec les autres; prendre pour argent comptant les données des autres alors qu'elles résultent d'un canon descriptionnel totalement inadapté à sa propre approche; dissimuler sous un même « étiquetage verbal », un même vocable de « concept », des horizons de pertinence ne partageant rien. On le voit, il est plus aisé pour deux disciplines de se méprendre que de se comprendre. Encore n'avons-nous envisagé que le passage de l'infra-conceptuel aux données. Une même analyse serait à mener, avec la même rigueur, concernant le passage des données à leur interprétation. Quoi qu'il en soit, ce travail sur l'horizon de pertinence, quoique trop rapide, a permis de mieux cerner les probables exigences d'une interdisciplinarité « focalisée ».

\section{Références}

Bateson G., 1972, Steps to an ecology of mind, Chicago, University of Chicago Press, 2000.

Charaudeau P., 2010 , « Pour une interdisciplinarité « focalisée » dans les sciences humaines et sociales », Questions de communication, 17, pp. 195-222.

Jauss H.-R., 1978, Pour une esthétique de la réception, trad. de l'allemand par C. Maillard, Paris, Gallimard, 1990.

Le Moigne J.-L., 1995, Les épistémologies constructivistes, Paris, Presses universitaires de France.

Leleu-Merviel S., 2003, « Les désarrois des « Maîtres du sens à l'ère du numérique » », pp. 17-34 in: Balpe J.-P., Saleh I., dirs, Hypertextes, hypermédias : créer du sens à l'ère numérique, Paris, Lavoisier/Hermès Science Publications.

Morin E., 1994, La complexité humaine, Paris, Flammarion.

Mugur-Schächter M., 2006, Sur le tissage des connaissances, Londres/Paris, Hermès/ Lavoisier.

- 2009, L'infra-mécanique quantique. Une révolution épistémologique révélée dans les descriptions de microétats, Paris, Éd. Dianoïa. 
S. Leleu-Merviel

Proust J., 2003, Les animaux pensent-ils?, Paris, Bayard.

Rastier J., 2003, « De la signification au sens. Pour une sémiotique sans ontologie », Texto! Accès : http ://www.revue-texto.net/Inedits/Rastier/Rastier_Semiotique-ontologie.html. Sperber D., Wilson D., 1989, La pertinence, Paris, Éd. de Minuit. 